\title{
Using Special Filter with Membership Function in Biomass Combustion Process Control
}

\author{
Jana Mižáková ${ }^{1}$, Ján Pitel ${ }^{2, *(\mathbb{D})}$, Alexander Hošovský ${ }^{2}$, Martin Kolarčík ${ }^{2}$ and \\ Madhawa Ratnayake ${ }^{2}$ \\ 1 Department of Natural and Humanities Sciences, Faculty of Manufacturing Technologies with a seat \\ in Prešov, Technical University of Košice, Prešov 08001, Slovakia; jana.mizakova@tuke.sk \\ 2 Department of Industrial Engineering and Informatics, Faculty of Manufacturing Technologies with a seat \\ in Prešov, Technical University of Košice, Prešov 08001, Slovakia; alexander.hosovsky@tuke.sk (A.H.); \\ martin.kolarcik@tuke.sk (M.K.); m.ratnayake1@gmail.com (M.R.) \\ * Correspondence: jan.pitel@tuke.sk; Tel.: +421-905-241605
}

Received: 6 July 2018; Accepted: 30 July 2018; Published: 1 August 2018

Featured Application: The filter with membership function can be applied in such devices where signals from the sensors contain strong disruptive (stochastic) parts. These components make it difficult to signal an acquisition process, complicate the monitoring and evaluation of the measured values, and often prevent the process control based on such a signal. Such a filter was successfully implemented in heating process control systems and it was proved that, for example, in biomass combustion control it was very effective at reducing random signal interferences caused by the use of the frequency converters for fan speed control.

Abstract: The paper deals with the special filtration method using a filter with membership function. The paper presents a model of a filter, its specific characteristics and some parameters that have an impact on quality of filtration. A filter with different membership functions (Gauss, Bell, Power and Triangle) was designed and tested for specific demands, which followed from the experience with the realization of a biomass combustion control system. Data obtained from the combustion process were extremely noisy (influenced by various transfer errors, disturbances and external interferences) and, therefore, had to be properly filtered. The paper also describes some results of filter simulation in the Matlab Simulink environment and its implementation into an on-line monitored process control system of biomass combustion. It was proven by implementation that such a filter can be useful for the signal filtering of oxygen concentration and carbon monoxide emission sensing and it can be very useful in reducing signal interferences arising in biomass combustion.

Keywords: biomass combustion; process control; filtering; membership function

\section{Introduction}

Biomass and especially woodchips is fuel with very unstable composition (varying moisture content in wood, type and quality of wood) in comparison with fossil fuels (natural gas, oil), and considering its heterogeneous characteristics it is necessary to control the amount of combustion air during fuel supply into the boiler furnace and also during the combustion phase [1,2]. If the amount of air is less than optimal, incomplete combustion occurs and the flue gas contains combustible components. On the other hand, in the case of supplying a large amount of combustion air, an energy loss (called flue loss) occurs. There is also a necessity to divide the supplied combustion air into primary air and secondary air [2,3]. Besides that, it is necessary to provide a high enough combustion temperature and time to complete the biomass combustion process. So there is a need to achieve 
simultaneously controlled values of the required boiler heat output and optimal conditions for the combustion process from both the efficiency and emissions point of view [4-6]. Therefore, the operation of biomass-fired boilers has to be properly controlled using a classical cascade [7] and also advanced control algorithms and methods [8,9] with the possibility of on-line system monitoring [10].

In medium-scale biomass-fired boilers, the amount of combustion air is in standard circumstances controlled by means of a Lambda probe that measures oxygen $\left(\mathrm{O}_{2}\right)$ concentration in a flue gas. In order to increase combustion efficiency and simultaneously to increase the amount of heat produced and to decrease harmful emissions, new control algorithms were designed based on information not only about $\mathrm{O}_{2}$ concentration in the flue gas but also about the trend of carbon monoxide (CO) emissions. These algorithms were implemented into the control systems of the biomass-fired boiler with the aim of reaching complete combustion with minimum excess of combustion air. It was proven that a low-cost wide band Lambda probe and simple CO sensor can be used for cost-effective biomass combustion control in medium-scale and, after some simplification, also in small-scale biomass-fired boilers [11-13]. However, due to strong interference in one type of boiler, the task of how to define a trend function of measured variables (especially $\mathrm{CO}$ emissions and $\mathrm{O}_{2}$ concentration in flue gas) had to be solved. Another problem that arose was how to correctly use the noisy measured data for biomass combustion control because it was difficult to apply in a process control system [14]. The measured values were influenced by various transfer errors, disturbances and external interferences and for that reason the measured data had to be appropriately smoothed and filtered.

There are many types of digital filters that can be used for signal filtration [15,16]. One of the interesting examples of digital filters is the infinite impulse response (IIR) filter. This filter consists of feedforward and feedback and it is also called the auto regressive moving average (ARMA) filter. A special group of digital filters is a group of finite impulse response (FIR) filters, also called moving average (MA) filters [17-19]. A filter that exhibits the properties of superposition, homogeneity, and shift invariance is called a linear time invariant (LTI) filter [20]. Its advantage is that one-dimensional LTI systems can be described by linear differential equations with constant coefficients.

Some of filters mentioned above are not convenient for biomass combustion signal filtering, because stochastic elements have an influence on the output of the filter (recursive IIR filters). For example, MA filters with exponential forgetting are not useful due to the algorithm of weight-counting [21]. The others are useful only with a mathematical model of the system (e.g., Kalman filter) [22].

\section{Membership Function Filter}

\subsection{Demands on the Filter}

Firstly, it must be emphasized that the supposed values of measured variables are predetermined (they are in known intervals) and it is necessary to appropriately eliminate extremely different values which arise from random interference during signal transmission. In the case of a long-lasting trend of measured values out of a supposed interval (for example, considerable increasing or decreasing of a measured variable) values are not eliminated. So the basic demand on the filter is that random changes would not have an effect on the filter output and, therefore, on controlled variables. Long-lasting changes out of the supposed interval must have an influence on the filter output and must be signified on the trend of measured variables.

Further reasons for the design of the specific filter are:

(1) Considering a filter application in digital controllers with limited computing capacity, the algorithm of the proposed filter has to be simple mathematically and relatively easily programmable.

(2) Using classical approximation methods by linear or non-linear trend functions is problematic, considering the huge number of random values in measured function behaviors as a result of strong interferences. 
(3) Using typical known filters (for example the Kalman filter) is questionable, considering that mathematical description of the measured variables behavior is unknown.

For these reasons, a filtering method using a weighted moving average was chosen with weight calculation on the basis of different membership functions.

\subsection{Description of the Filtering Method}

The membership function filter, as it was named, is similar to the weight moving average filter, which is counted from $k$ measured values, but weights are calculated based on the membership function for belonging to a set of $k$ measured values. This is the most important difference from a weighted moving average filter, because if a set of measured values has changed, weights also change due to their dependence on an average set of values. Mathematically, such a filter can be expressed as follows.

The sample of the non-filtered signal $u\left(t_{i}\right)$ includes deterministic $u_{d}\left(t_{i}\right)$ and stochastic $u_{s}\left(t_{i}\right)$ elements:

$$
u\left(t_{i}\right)=u_{d}\left(t_{i}\right)+u_{s}\left(t_{i}\right)
$$

The stochastic part of the signal can be suppressed by the substitution for the counted moving weight average from the last $k$ samples of the non-filtered signal:

$$
\bar{u}\left(t_{i}\right)=\frac{\sum_{j=1}^{k} u\left(t_{j}\right) \cdot w_{j}}{\sum_{j=1}^{k} w_{j}}
$$

where $j=1, \ldots, k$ and $k$ is the chosen number of samples, $u\left(t_{j}\right)$ are last $k$ signal samples which contain the deterministic and stochastic element, and $w_{j}$ are counted weights from interval $(0-1\rangle$ for $k$ last obtained signal samples.

The principle diagram of such a membership function filter for $k$ samples of the non-filtered signal is in Figure 1. It consists of block 1 for sampling of the non-filtered signal, block 2 for sampling of the filtered signal, blocks 3 for weight assignment of non-filtered samples according to selected membership function by block 7, and blocks 4 (multiplication), 5 (summation) and 6 (division) for calculation of the filter output according to Equation (2). The input to the filter is the current sample of the non-filtered signal containing deterministic and stochastic parts. For this sample and also for previous $k-1$ stored samples, weights are calculated according to selected membership function and multiplied by used samples. In the next step, the sum of products and the sum of weights are calculated in order to be divided, and the new filtered sample is obtained from the filter output. Finally, the output of the filter is saved for weight counting in time $t_{i+1}$. Due to division in the output block of the filter, there is a condition for filter implementation that the sum of weights can be never equal to zero.

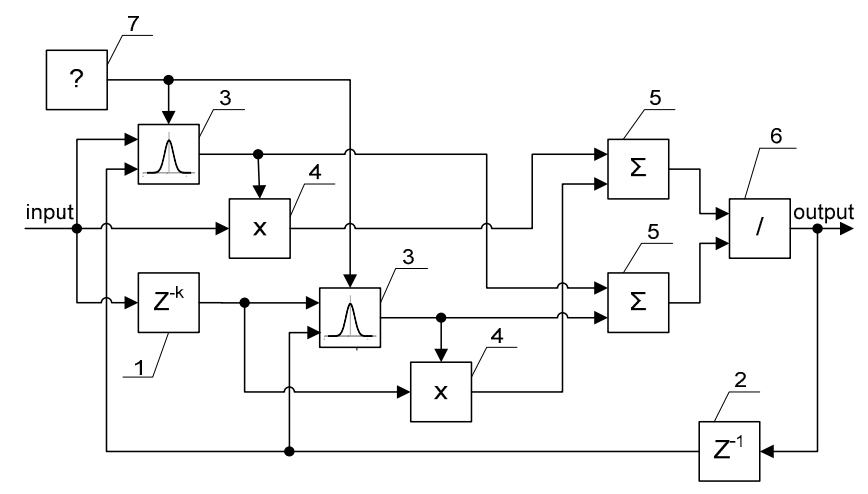

Figure 1. Basic principle of filter operation for $k$ samples of the non-filtered signal. 


\subsection{Membership Function and Its Weights Calculus}

The membership function of several different distribution functions can be chosen as shown in Figure 2. For a normal distribution function (Gauss function), the Equation is:

$$
f(x)=\frac{1}{\sigma \sqrt{2 \pi}} e^{-\frac{(x-\mu)^{2}}{2 \sigma^{2}}}
$$

After some simplifications this can be derived for weight calculation:

$$
w(x)=e^{-\frac{(x-\mu)^{2}}{s}}
$$

where $s$ is defined as sensitivity and $\mu$ is last weighted average.

Similarly, for the Triangle function:

$$
w(x)=1-\left|\frac{x-\mu}{s}\right|
$$

Power function:

$$
w(x)=1-\left|1-\frac{x}{\mu}\right|^{s}
$$

and Bell function:

$$
w(x)=\frac{1}{1+\left|\frac{x-\mu}{s}\right|^{2}}
$$

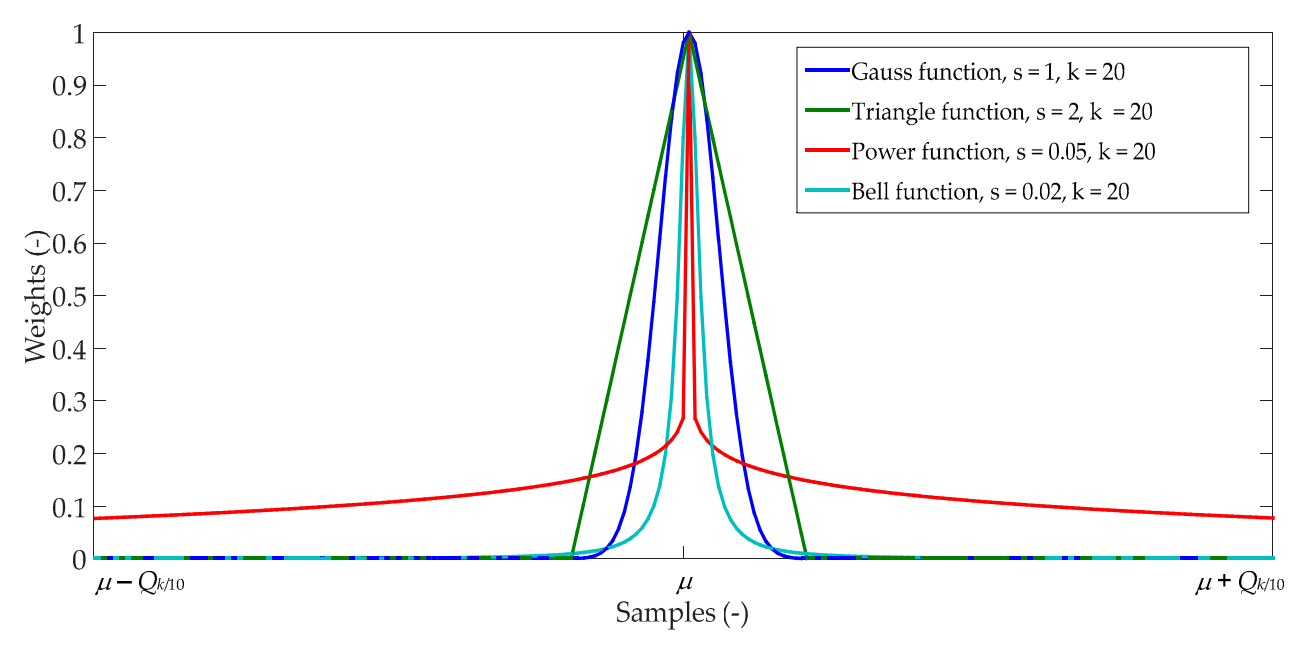

Figure 2. Examples of membership functions.

\subsection{Filter Parameters}

Operation of the membership function filter depends mainly on two parameters: sensitivity $s$ of the membership function and the number of samples $k$ stored in the filter. In Figures 3-6, a comparison of various sensitivity $s$ is shown for different membership functions with weight calculations according to Equations (4)-(7).

In Figure 3, there are weights calculated according to Gauss function. Referring to Figure 3, by filtering using Equation (4), samples which are closer to average $\mu$ have an assignment of higher weights and only samples further away from the average $\mu$ have an assignment of very low weight. If the sensitivity coefficient $s$ is lower, the interval of higher weights will be narrower, i.e. only samples which are very near to average $\mu$ have an assignment of high weights. The Triangle function in 
Equation (5) is also suitable for using as a membership function, because as can be seen in Figure 4 it has a similar results of weights assignment as the Gauss or Bell functions, but does not have a concave shape near average $\mu$. The Power function in Equation (6) is not entirely appropriate for filtering because the function has the shape of a tip so the higher weights are assigned at a very narrow interval (Figure 5). The Bell membership function with weights in Equation (7) has a similar behavior (Figure 6) to the Gauss function. The main difference is the smaller width of the interval with higher weights, which seems to be useful for filtering slowly changing waveforms.

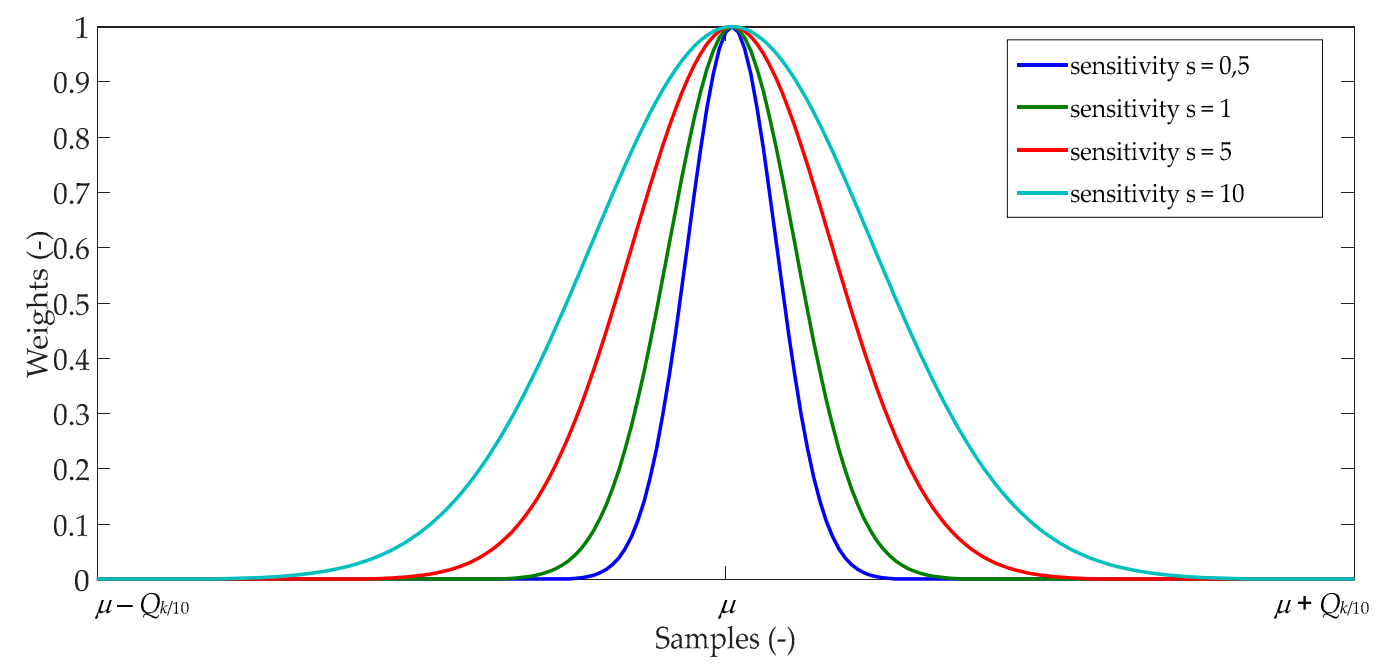

Figure 3. The assignment of weights by using the function in Equation (4) as the membership function.

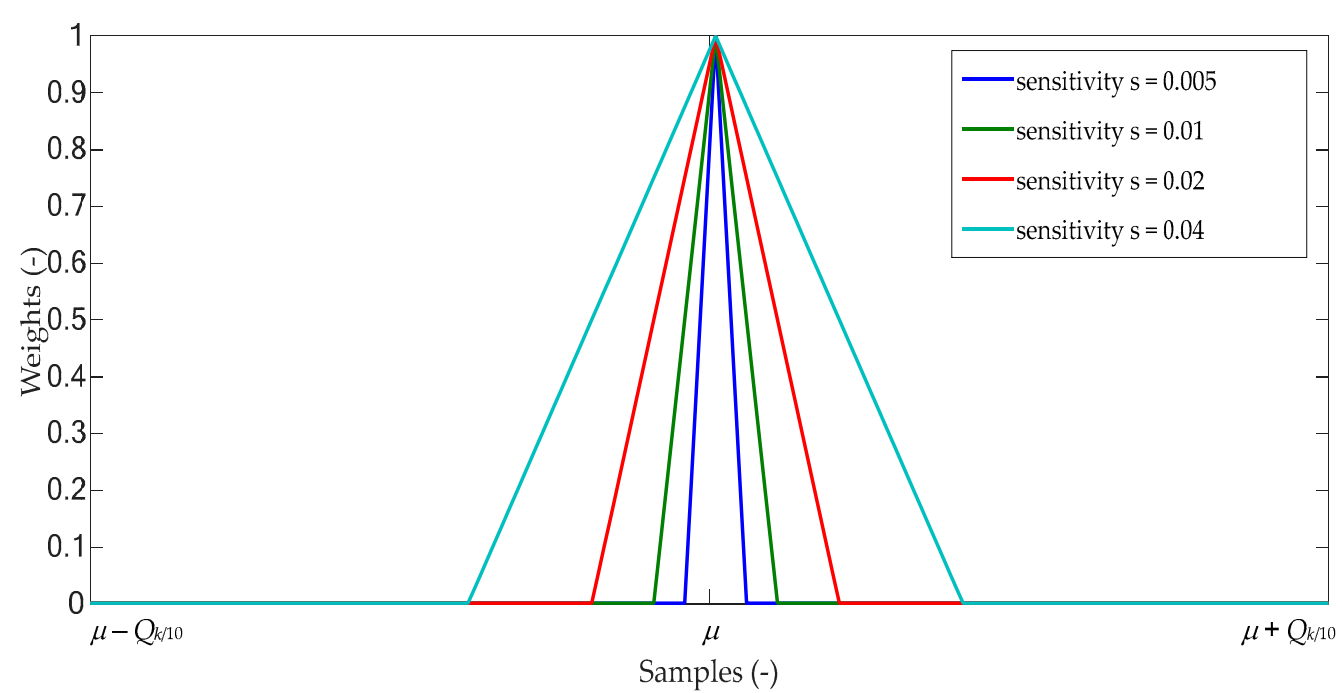

Figure 4. The assignment of weights by using the Triangle function in Equation (5) as the membership function. 


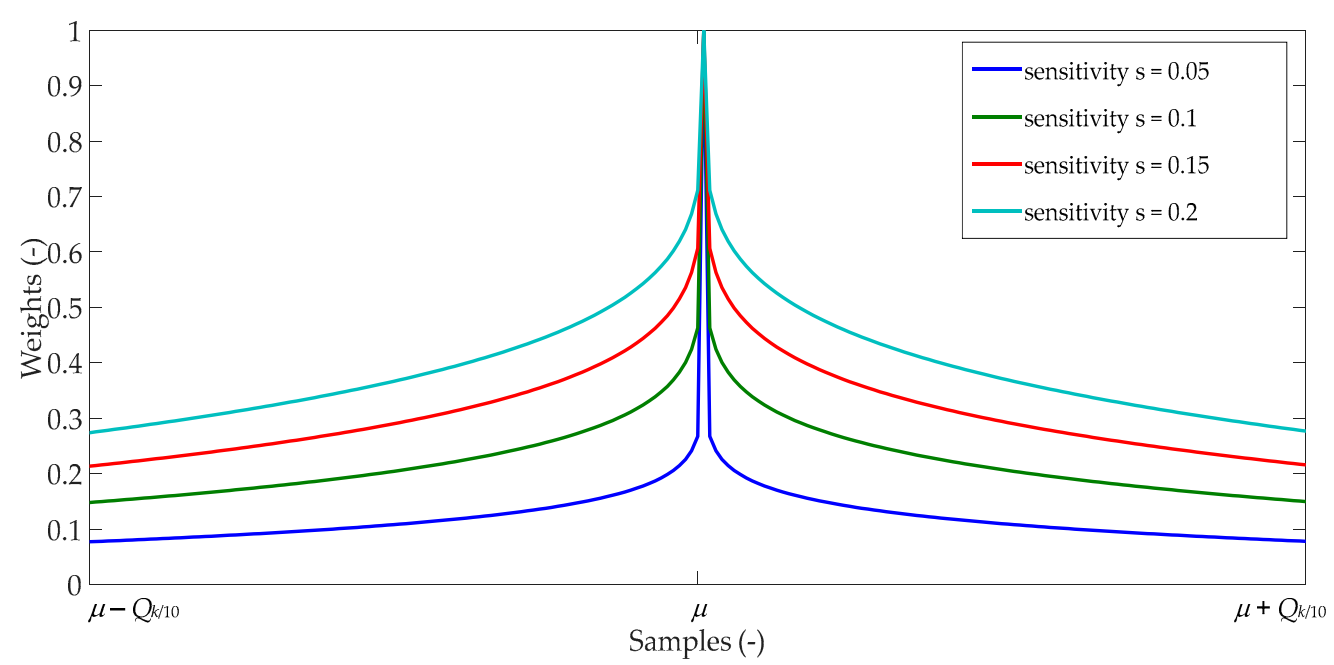

Figure 5. The assignment of weights by using the Power function in Equation (6) as the membership function.

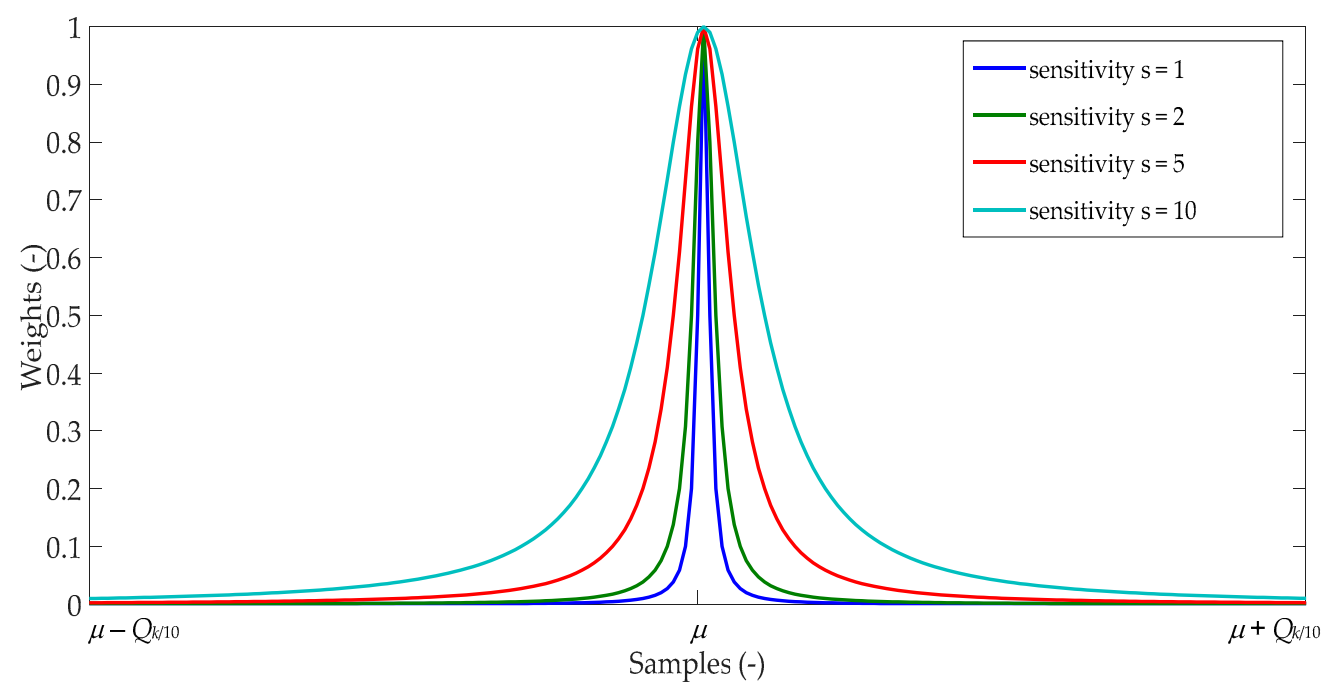

Figure 6. The assignment of weights by using the Bell function in Equation (7) as the membership function.

\subsection{Testing of the Membership Function Filter}

The model of the designed filter with the membership function was created in $C$ programming language (Microsoft Visual C++ Express 2008 for Matlab R2007A, Microsoft Corporation, Redmond, WA, USA, 2010) and it was implemented into the Matlab Simulink (Matlab R2010a, The MathWorks Inc., Natick, MA, USA, 2010) environment as an individual block. Firstly, the model time responses to unit step were simulated for $k=20$ samples using different membership functions (Figure 7). 


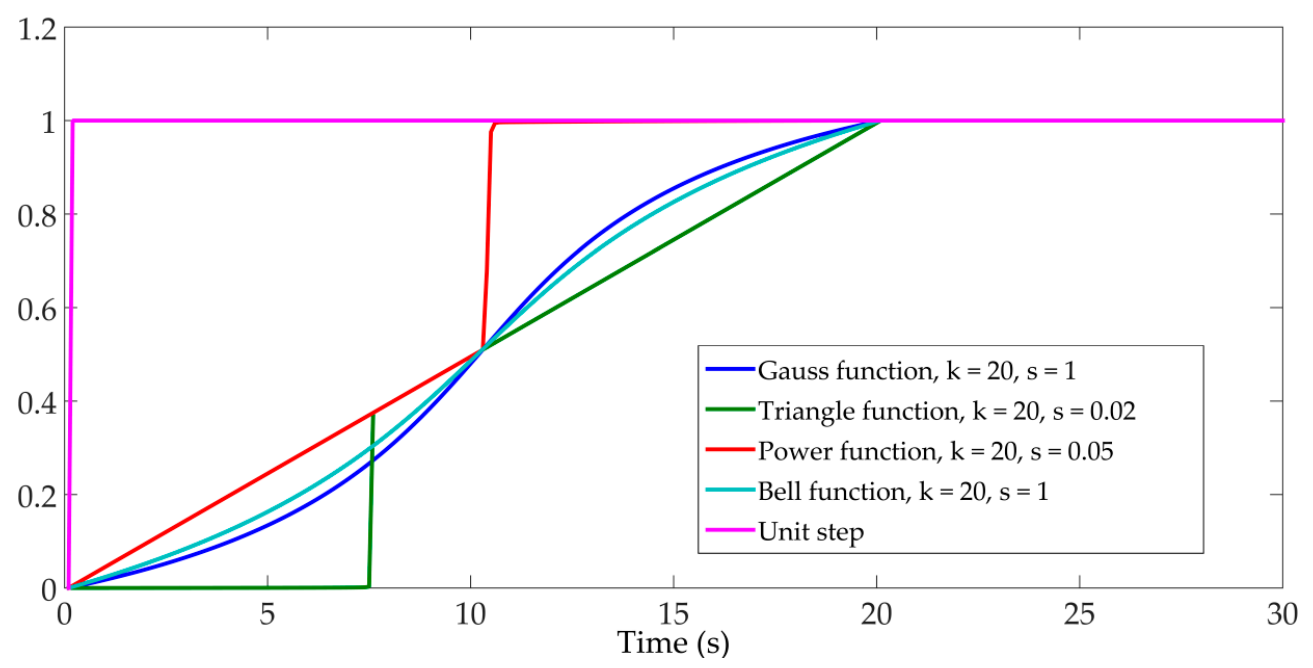

Figure 7. Filter time responses to unit step for various membership functions.

Next, the model was simulated for filtration of the sine function with random noise (Figure 8) again for $k=20$ samples using different membership functions with the following sensitivity parameters: for the Gauss function in Equation (4) $s=1$, for the Triangle function in Equation (5) $s=0.02$, for the Power function in Equation (6) $s=0.05$, and for the Bell function in Equation (7) $s=1$.

The influence of sensitivity $s$ and the number of samples $k$ were tested on a filter with the Gauss membership function. Filter time responses to unit step for different number of samples $k$ are in Figure 9. The change of parameter $k$ can be seen in Figure 10 as the time delay of filtration of the noisy measured temperature. The change of the membership function sensitivity $s$ as influenced on the filter output can be seen in Figure 11.

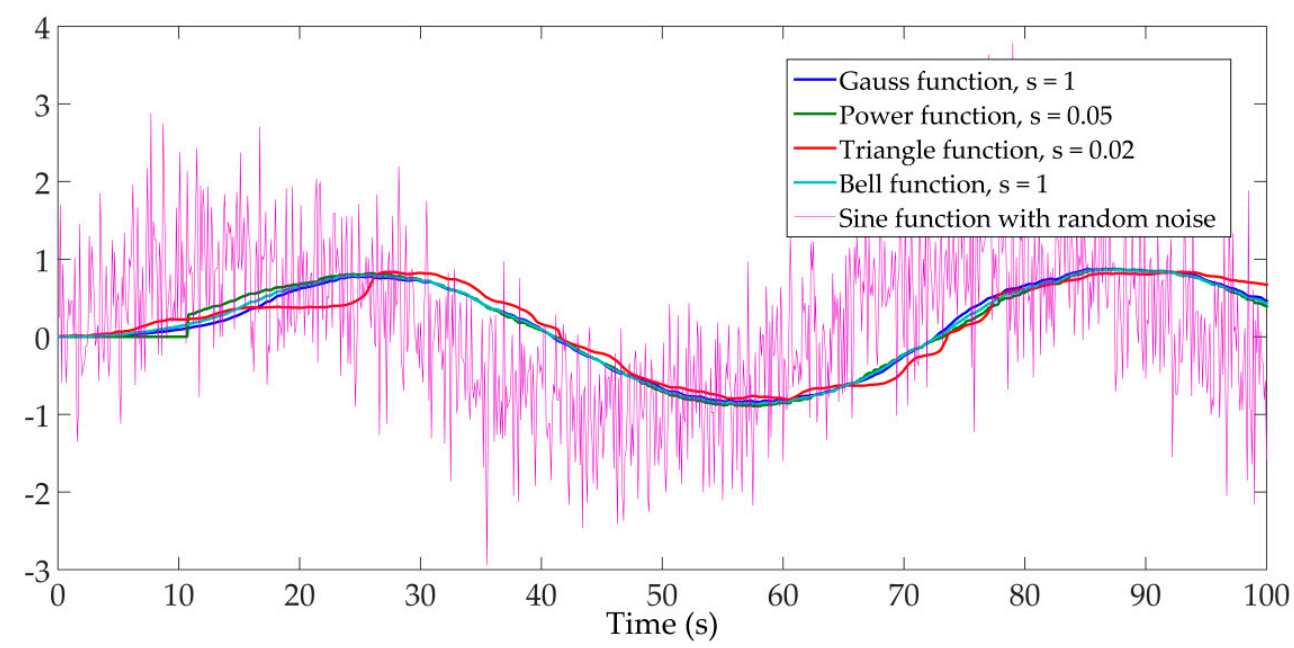

Figure 8. Comparison of using different membership functions for filtration of sine function with random noise. 


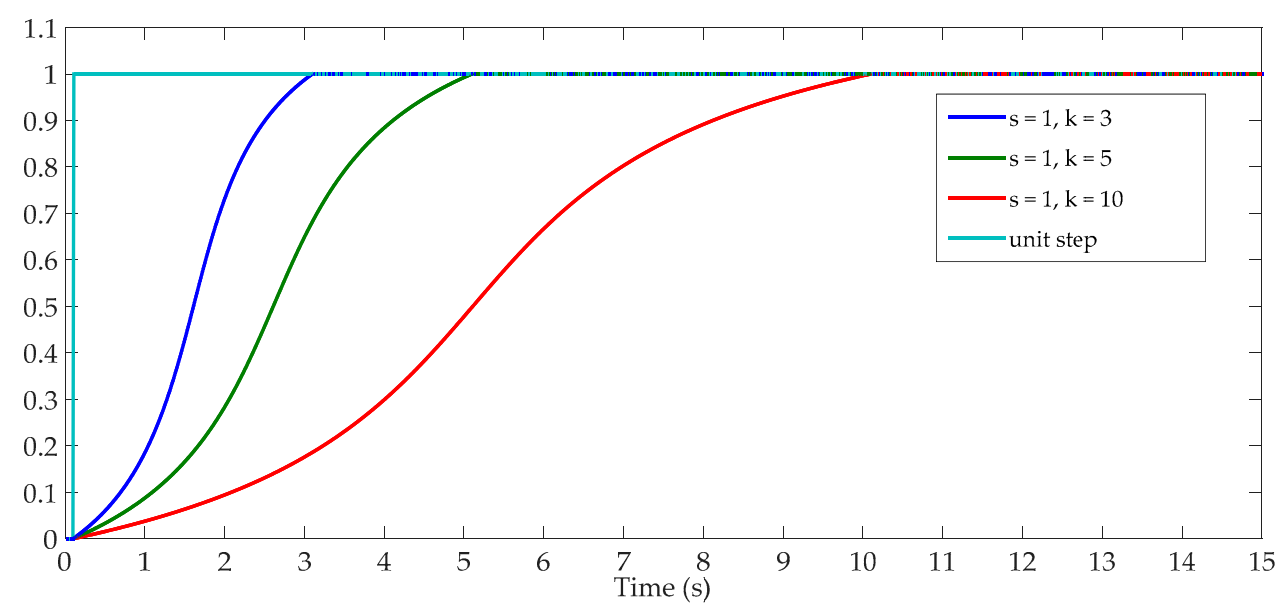

Figure 9. Filter time responses to unit step for different number of samples.

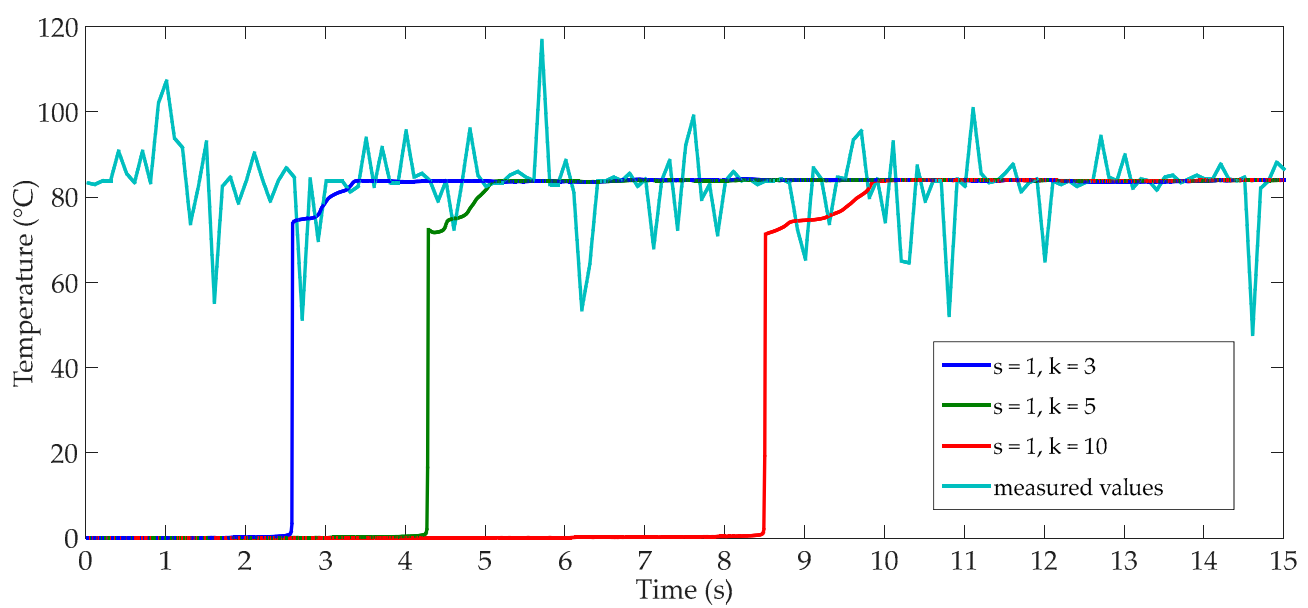

Figure 10. Filtering of the noisy measured variable for different number of samples.

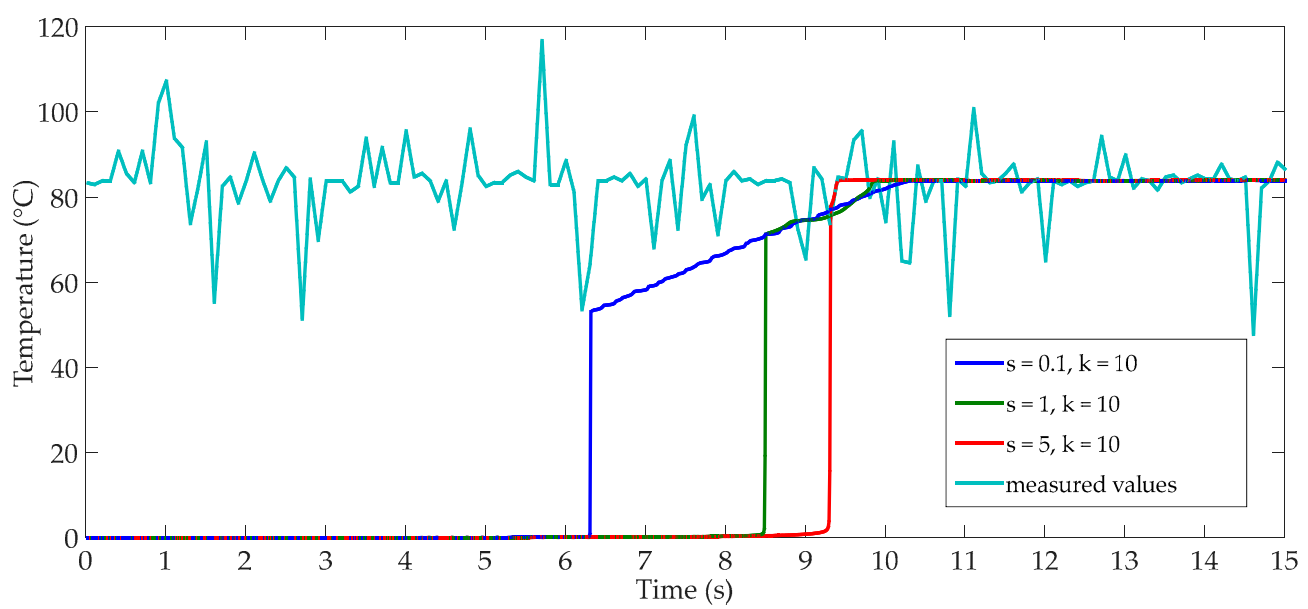

Figure 11. Filtering of the noisy measured variable for different sensitivity of the membership function.

\section{Results}

The designed filter with the Gauss membership function was implemented into a free programmable industrial process control system of biomass combustion which was monitored on-line by a Supervisory Control and Data Acquisition (SCADA) system [11]. On-line monitoring allows 
visualization of the technological process (graphical schemes, diagrams, trends and reports), to evaluate the quality of combustion process and to change the control parameters with access via the internet [1]. The designed control and monitoring system was installed for the control of two medium-scale biomass-fired boilers for woodchip combustion (with different powers and different manufactures). Grate furnace combustion technology was used in both boilers, and the sketch of one type of boiler is in Figure 12. The reversible shifting grate is situated in the firebox, the primary combustion air is fed by means of a fan under the grate, the secondary one above the grate. The amount of primary and secondary air is controlled by means of corresponding fan rpm [3].

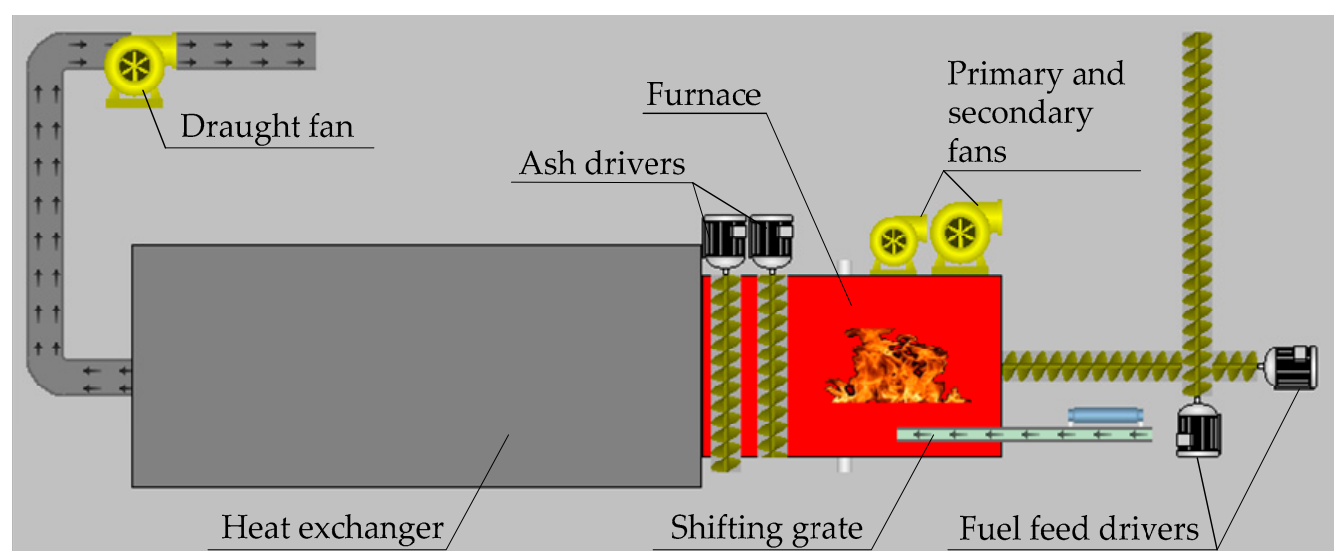

Figure 12. The sketch of $600 \mathrm{~kW}$ biomass-fired boiler.

In Figure 13 there is an example of the process variables' remote monitoring. As can be seen, some of them are interfered with strongly. In particular the quality of $\mathrm{O}_{2}$ concentration sensing (yellow course in Figure 13) was unable to be used for effective control of the combustion process. The correct values of $\mathrm{O}_{2}$ concentration in the flue gas are very important for the combustion process control because efficiency of the biomass combustion depends on the excess air ratio $\lambda$, which can be obtained from the measured $\mathrm{O}_{2}$ concentration as follows [23]:

$$
\lambda=\frac{21}{21-\mathrm{O}_{2} \%}
$$

where $\mathrm{O}_{2} \%$ is oxygen concentration in the flue gas in percentage.

That is why, firstly, the new filter with a membership function was applied for filtering $\mathrm{O}_{2}$ concentration measured data and the result of filtration is shown in Figure 14.

The optimal range of excess air ratio $\lambda$ for biomass combustion is usually in the interval (1.4-2) and its optimal value depends on the type of wood, the moisture content in the wood, combustion chamber construction, and so on. However, the most optimal biomass combustion operating conditions are when the compromise between maximal combustion efficiency and minimal CO emissions is achieved. One of the control algorithm's tasks is to find such a value of excess air ratio $\lambda$ so that $\mathrm{CO}$ emissions would be minimal, even with the change in fuel parameters. To fulfill this task, it is necessary to continuously monitor a trend between $\mathrm{CO}$ emission and excess air ratio and, consequently, to change the set point of $\mathrm{O}_{2}$ concentration in the flue gas [11,24]. That is why this filter was also applied for filtering CO emissions' measured data, and the result of filtration is shown in Figure 15. 


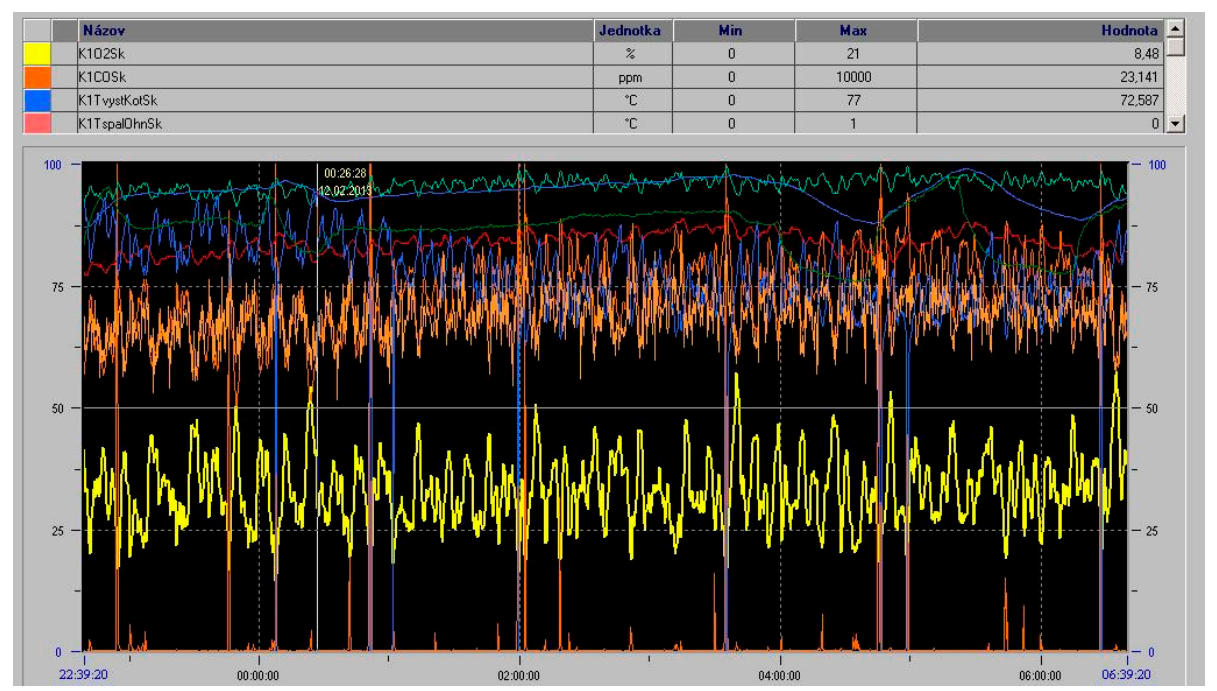

Figure 13. Remote monitoring of biomass combustion process variables.

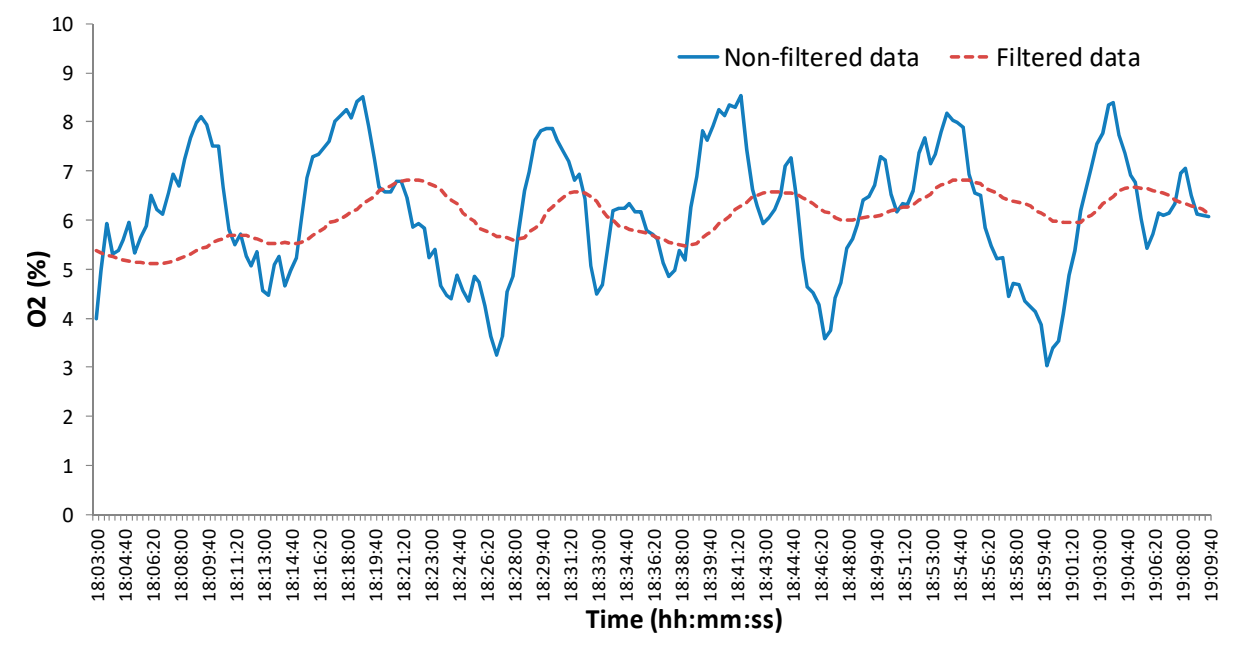

Figure 14. Filtering of $\mathrm{O}_{2}$ concentration.

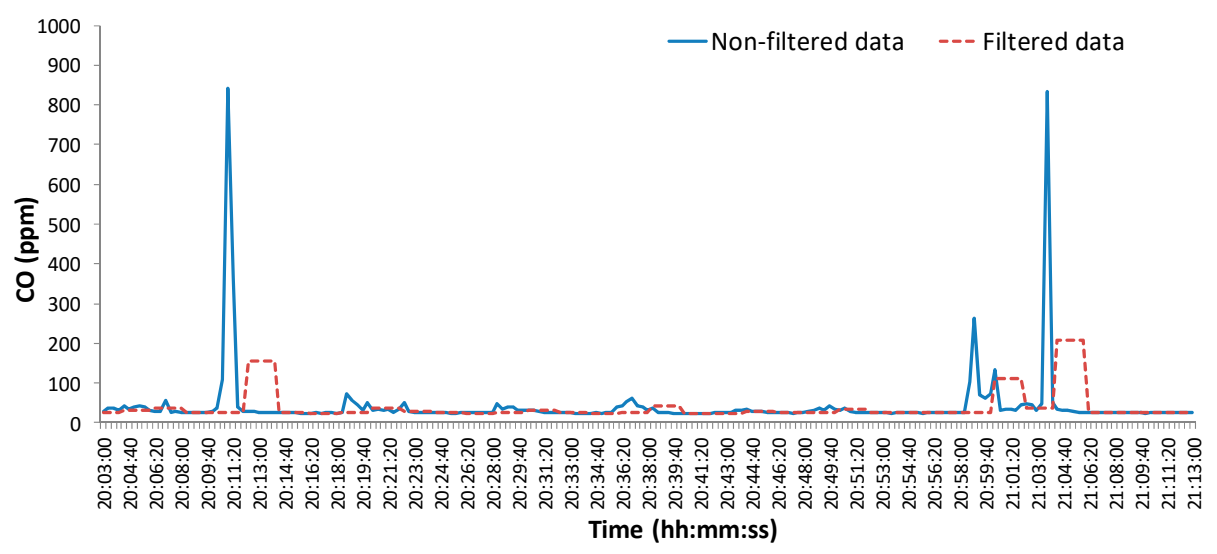

Figure 15. Filtering of $\mathrm{CO}$ concentration.

The quality of filtration has been confirmed by comparison of the membership function filter with the other types of filters. Figure 16, for example, shows a comparison with a moving average filter. As such, it can be seen that the moving average filter does not exclude values that are extremely 
different. On the other hand, the membership function filter monitors the trend of data, but there is a significant time delay. However, this time delay does not have an important impact on $\mathrm{O}_{2}$ or $\mathrm{CO}$ sensing in the biomass combustion process because the trend of their concentration is important not their current values.

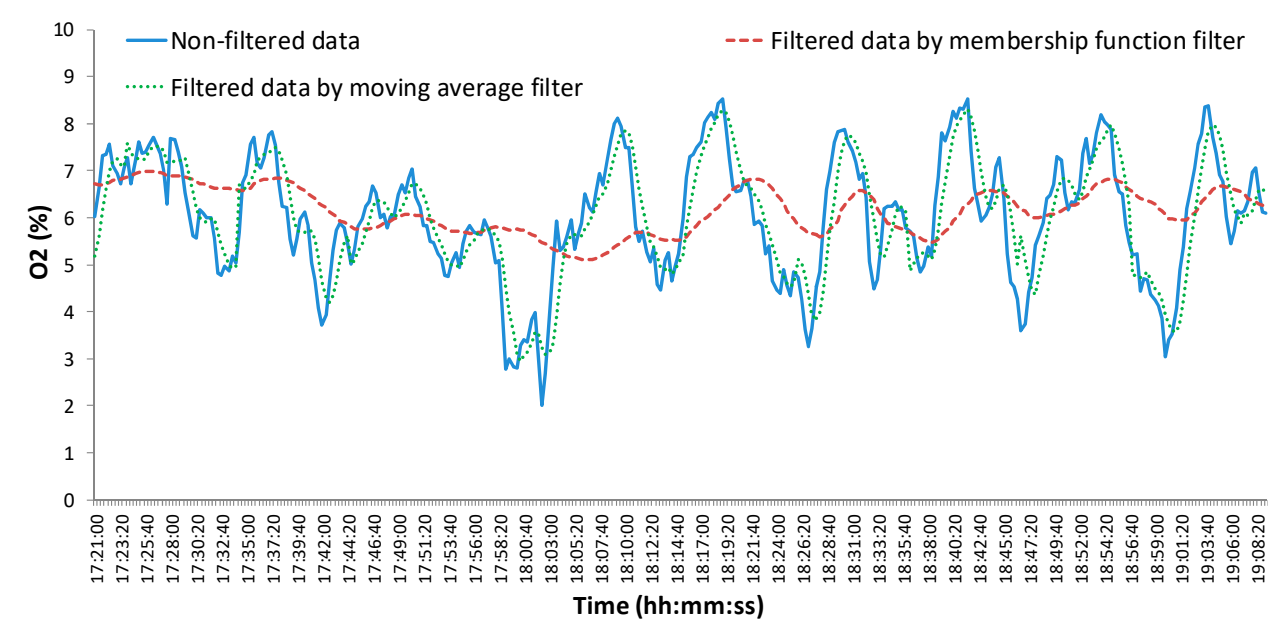

Figure 16. Comparison of membership function filter with moving average filter.

\section{Conclusions}

Behavior of the membership function filter can be influenced mainly by two parameters:

(1) Sensitivity $s$ of the membership function-this determines how strongly the samples near the average value are preferred, respectively, and how strongly the samples away from the average value are suppressed.

(2) The number of samples $k$ used for counting of moving weighted average-higher value of parameter $k$ causes a longer time delay of the filter, but on the other hand if $k$ is very low the filtered data copy more real data with noise.

The filter with a normal distribution membership function was chosen for filtering important variables in the biomass combustion process. This function with appropriately selected parameters $s$ and $k$ ensures that the remote values from the average have lower weights (in opposite to Bell and Power function) and values which are close to the average have concave shape of weights (in opposite to Triangle function).

This was proven by implementation of the designed special filter into a control system of biomass combustion process that it is useful for signal filtering of $\mathrm{O}_{2}$ concentration measured by a low-cost wide band Lambda probe and $\mathrm{CO}$ emissions measured by a simple $\mathrm{CO}$ sensor. The implemented filter based on the Gauss membership function with parameters set to values $k=60, s=4$ had a significant effect on reducing signal interferences arising in the biomass. The implemented control algorithms based on using information about the filtered tendency of nascent carbon monoxide successfully stabilized the combustion process in medium-scale biomass-based boilers, especially during the start of combustion or its interruption due to some disturbance. These advanced algorithms can also compensate for varying woodchip parameters (moisture content in wood, type and quality of wood), which is an object of intensive research at the authors' workplace [25].

\section{Patents}

Inventors: Boržíková, J., Mižák, J., Pitel', J., Židek, K. Membership Function Filter. Patent number: SK201200021-A3.

Inventors: Balara, M., Mižák, J., Mižáková, J., Pitel', J. Discrete Signal Weighting Average Computing Device. Patent number: SK201350050-A3. 
Author Contributions: Author J.M. dealt with theoretical filter analysis and she conceived of the filter description in the paper. Authors J.P. and A.H. implemented the filter in the biomass combustion process control and performed experiments. Authors M.K. and M.R. analyzed the data.

Funding: This research was funded by Agentúra na Podporu Výskumu a Vývoja grant number APVV-15-602.

Acknowledgments: This work was supported by the Slovak Research and Development Agency under the contract No. APVV-15-0602.

Conflicts of Interest: The authors declare no conflict of interest.

\section{References}

1. Hrdlička, J.; Šulc, B.; Plaček, V.; Vrána, S. Impact of control solutions on ecology and economy of small-scale biomass boilers. Int. J. Circuits Syst. Signal Process. 2011, 5, 247-254.

2. Pitel', J.; Boržíková, J.; Mižák, J. Biomass combustion process control using artificial intelligence technique. In Proceedings of the XXXV. Seminar ASR'010 Instruments and Control, Ostrava, Czech Republic, 30 April 2010; pp. 317-321.

3. Skok, P.; Rimár, M.; Mižák, J. Woodchip combustion process quality and the amount of combustion air. Appl. Mech. Mater. 2013, 308, 115-119. [CrossRef]

4. Hrdlička, J.; Šulc, B.; Vrána, S.; Plaček, V. Ecological aspects of small-scale biomass combustion control. In Proceedings of the Develepment, Energy, Environment, Economics (DEEE '10), Puerto De La Cruz, Spain, 30 November-2 December 2010; pp. 388-393.

5. Mullerová, J.; Hloch, S.; Valíček, J. Reducing emissions from the incineration of biomass in the boiler. Chemické Listy 2010, 104, 876-879.

6. Oswald, C.; Plaček, V.; Šulc, B. Advanced control with economic-ecological optimization for biomass-fired boilers. In Proceedings of the 2014 15th International Carpathian Control Conference (ICCC 2014), Velke Karlovice, Czech Republic, 28-30 May 2014; pp. 407-412.

7. Tóthová, M.; Balara, M.; Dubják, J. Simulation model of cascade control of the heating system. Int. J. Eng. Res. Afr. 2015, 18, 20-27. [CrossRef]

8. Hošovský, A. Biomass-fired boiler control using simulated annealing optimized improved Varela immune controller. Acta Polytechnica Hungarica 2015, 12, 23-39.

9. Vrána, S.; Plaček, V.; Oswald, C.; Šulc, B.; Neuman, P. Neural network evaluation of combustion process for continuous control of small scale biomass fired boilers. In Proceedings of the 19th IFAC World Congress on International Federation of Automatic Control (IFAC 2014), Cape Town, South Africa, 24-29 August 2014; pp. 1440-1445.

10. Boržíková, J.; Pitel', J.; Mižák, J. Monitoring of operational states of biomass combustion process. Appl. Mech. Mater. 2013, 308, 153-158. [CrossRef]

11. Pitel', J.; Mižák, J. Cost effective biomass combustion process control. In Proceedings of the 8th PP\&PSC (Power Plant and Power System Control) IFAC Symposium, Toulouse, France, 2-5 September 2012; pp. 616-620.

12. Pitel', J.; Mižáková, J. Implementation of filter with membership function into control system of biomass combustion process. In Proceedings of the 19th International Conference on Systems (part of CSCC'15), Zakynthos Island, Greece, 16-20 July 2015; pp. 388-392.

13. Pitel', J.; Mižáková, J.; Hošovský, A. Biomass combustion control and stabilization using low-cost sensors. Adv. Mech. Eng. 2013, 5, 1-7. [CrossRef]

14. Placier-Zavala, E.; Plaček, V. Filtering of data specific for biomass combustion. Proceedings of Seminar ASR '2012 Instruments and Control, Ostrava, Czech Republic, 27 April 2012; pp. 83-88.

15. Madisetti, V.K. The Digital Signal Processing Handbook, 2nd ed.; CRC Press: Boca Raton, FL, USA, 2010.

16. Sovka, P.; Čmejla, R.; Šmejkal, L. What about... digital filters I. Automation 2005, 48, 7-8.

17. Chalupa, P.; Novák, J.; Januška, P. State space MPC using state observers. Int. J. Circuits Syst. Signal Process. 2014, 8, 9-14.

18. Mastorakis, N.E. New general transformations for 2-D FIR and IIR filters' design. WSEAS Trans. Circuits Syst. 2011, 10, 1-9.

19. Tan, L. Digital Signal Processing: Fundamentals and Applications; Academic Press: New York, NY, USA, 2011.

20. Williams, C.S. Designing Digital Filters; Prentice-Hall: Upper Saddle River, NJ, USA, 1986. 
21. Boržíková, J.; Mižák, J.; Tóthová, M.; Židek, K. Using the weight moving average for filtering of measured data. In Proceedings of the Automation in Theory and Practice (ARTEP 2012), Stará Lesná, Slovak, 22-24 February 2012.

22. Židek, K.; Saloky, T.; Maxim, V. Global navigation and its specification using Kalman filter. Transf. Inovácií 2006, 9, 159-162.

23. Pitel', J.; Mižák, J. Computational intelligence and low cost sensors in biomass combustion process. In Proceedings of the 2013 IEEE Symposium on Computational Intelligence in Control and Automation (CICA), Singapore, 16-19 April 2013; pp. 165-168.

24. Eskilsson, D.; Quicklund, H.; Johansson, M.; Eliasson, T.; Österberg, S. Development of a Control System That Utilises the Information from Gas Sensors to Control the Supply of Combustion Air; Sveriges Provnings-och Forskningsinstitut Energiteknik: Borås, Sweden, 2004.

25. Pitel', J.; Mižák, J. Approximation of CO/Lambda biomass combustion dependence by artificial intelligence techniques. In Proceedings of the 22nd International DAAAM Symposium, Vienna, Austria, 23-26 November 2011; pp. 0143-0144.

(C) 2018 by the authors. Licensee MDPI, Basel, Switzerland. This article is an open access article distributed under the terms and conditions of the Creative Commons Attribution (CC BY) license (http://creativecommons.org/licenses/by/4.0/). 